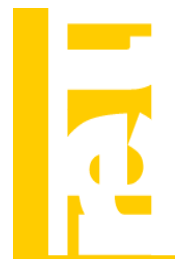

\title{
SATISFACCIÓN CON LA CONCILIACIÓN LABORAL Y FAMILIAR DE LOS ASALARIADOS EN ESPAÑA
}

\author{
Paz Rico Belda ${ }^{1}$ \\ Universidad de Valencia
}

Recibido 7 Diciembre 2011; aceptado 3 Octubre 2012

\section{Resumen}

El objetivo del presente estudio es identificar los factores determinantes del nivel de satisfacción con la conciliación laboral y familiar de los asalariados en España, haciendo especial hincapié en las diferencias de género y de nacionalidad. Para ello se estima un modelo de respuesta múltiple ordenada, utilizando datos de la Encuesta de Calidad de Vida en el Trabajo, para el año 2010. Los resultados obtenidos muestran que, después de controlar por las características personales y laborales de los asalariados, no se evidencia la existencia de efectos diferenciales en la satisfacción con la conciliación laboral y familiar ni por razón de género ni de nacionalidad. Un análisis más detallado permite concluir que los factores que explican no detectar efecto diferencial por nacionalidad están relacionados con las características personales y con el tiempo en el trabajo, mientras que el no obtener efecto diferencial por género se debe a la inclusión de variables relacionadas con las características personales y con la conciliación.

Palabras clave: Satisfacción laboral, conciliación, diferencia de género, diferencia por nacionalidad, modelos de respuesta múltiple ordenada.

Clasificación JEL: J28, C25.

\begin{abstract}
This study aims to identify the explanatory factors of satisfaction in conciliation between career and familiar life of employees in Spain, with special emphasis on gender and nationality differences. For doing that, an ordered multiple answer model is estimated, using data from the Living Standards Quality in the Job, corresponding to year 2010. The results show that, after controlling by personal and employment characteristics of employees, there are no differences by nationality or by gender in satisfaction in conciliation between career and familiar life. A more detailed analysis allows conclude that the inclusion of variables in relation with personal characteristics and working hours explains that there is not nationality difference, while personal characteristics and conciliation variables they explain that gender difference doesn't exist.
\end{abstract}

Key words: Job satisfaction, conciliation, gender differences, nationality differences, ordered multiple answer models.

JEL Classification: J28, C25.

\footnotetext{
1 Dirección de contacto: paz.rico@uv.es. Quisiera agradecer los comentarios y sugerencias de dos evaluadores anónimos y del editor, que han permitido una mejora sustancial del artículo. No obstante, cualquier error sólo es responsabilidad mía.
}

(C) Revista de Economía Laboral 


\section{Introducción}

La creciente incorporación de la mujer al mercado laboral ha provocado importantes transformaciones sociales y ha puesto en el punto de mira del debate social y académico la conciliación entre la vida laboral y familiar. La conciliación representa uno de los principales retos políticos en Europa y, muy particularmente, en España puesto que, desde una perspectiva comparada, es uno de los países de Europa en los que las políticas públicas de apoyo a las familias son más débiles (Gracia y Bellani (2010)).

La conciliación laboral y familiar tiene como objetivo mejorar la calidad de vida de los trabajadores, al permitir hacer compatible las distintas facetas de su vida: empleo, familia y tiempo personal. La conciliación laboral y familiar requiere la participación de todos los agentes sociales, en general, y de las empresas, en particular. En los últimos años, cada vez más empresas se muestran dispuestas a mejorar la calidad de vida de sus plantillas, puesto que finalmente ello revierte en su propio beneficio (Ponce 2007). Las dificultades de no poder compatibilizar vida laboral y familiar no sólo tiene repercusiones negativas en la persona trabajadora, también tiene consecuencias negativas para las empresas al aumentar el absentismo laboral y disminuir la productividad ${ }^{2}$. Por tanto, cada vez más la conciliación laboral y familiar se considera una herramienta básica de gestión de recursos humanos, puesto que permite maximizar las potencialidades de los trabajadores.

La literatura sobre los efectos que la conciliación tiene en la empresa, en el comportamiento de los trabajadores y en el bienestar familiar es muy extensa ${ }^{3}$, pero lo que no se ha analizado en demasía es el grado de satisfacción que muestran los trabajadores con la conciliación entre su vida laboral y familiar. La encuesta sobre la Calidad de Vida en el Trabajo (ECVT), que realiza el Ministerio de Trabajo e Inmigración, incluyó por primera vez en 2010 una pregunta en la que se solicitaba al entrevistado que declarase el grado de satisfacción con la conciliación entre su vida laboral y familiar. El propósito de este trabajo es explotar la información proporcionada por la ECVT, con el objetivo de conocer los factores que determinan el grado de satisfacción con la conciliación laboral y familiar de los asalariados en España, y si existen diferencias de género

\footnotetext{
${ }^{2}$ Ver los informes del Observatorio efr (2009,2010), Chinchilla y León (2011) y de IESE (2012).

3 Entre otros trabajos cabe citar los de Drew et. al. (1998), Moltó (2005), Ponce (2007), Lewis et al. (2008), European Commision Europea (2005, 2009), Vera-Martínez y Martín (2009), Cantera et al. (2009), Gracia y Bellani (2010) y IESE (2012).
} 
y de nacionalidad. Para ello se va a estimar un modelo de respuesta múltiple ordenada. Recientemente, IESE (2012) presenta los resultados de una encuesta sobre satisfacción con la conciliación y concluye que en España los hombres se muestran más insatisfechos que las mujeres con las medidas de conciliación de su empresa, mientras que los empleados con hijos se muestran más satisfechos que los empleados sin hijos. Asimismo, concluye que la satisfacción disminuye con la edad y aumenta con el tamaño de la empresa ${ }^{4}$.

La organización del trabajo es la siguiente. En el apartado 2 se analizan descriptivamente los datos utilizados. En el apartado 3 se presentan los resultados, y, finalmente, en el apartado 4 se recogen las principales conclusiones del trabajo.

\section{Datos}

Los datos utilizados en este trabajo proceden de ECVT del año $2010^{5}$. La muestra inicialmente seleccionada está compuesta por los trabajadores asalariados, que no superan la edad de 65 años. Se han excluido, por tanto, los empresarios, los trabajadores autónomos y las personas que trabajan en el negocio familiar. Después de eliminar las observaciones que no proporcionan información sobre alguna de las variables consideradas, el tamaño muestral asciende a 5.594 asalariados. De éstos el 44,8\% son mujeres y, por tanto, el 55,2\% de las observaciones corresponden a hombres. De esta muestra, los trabajadores con nacionalidad diferente a la española ascienden a 532 personas, el 9,5\% de las observaciones totales ${ }^{6}$.

En la ECVT se pide al trabajador entrevistado que determine cuál es su grado de satisfacción con la conciliación entre su vida laboral y familiar en base a una escala de 0 a 10 . No obstante, en este trabajo se han reagrupado los once valores de la escala original en tres $(0,1,2)$ obteniéndose la variable dependiente SAT de la siguiente forma ${ }^{7}$ : original)

- 0 para los trabajadores insatisfechos (respuestas 0 a 4 en la escala

- 1 para los trabajadores satisfechos $(5,6$ y 7$)$

\footnotetext{
${ }^{4}$ Los resultados se basan en la comparación de los valores medios muestrales.

5 Una descripción detallada del proceso de selección muestral se puede encontrar en el enlace http://www.mtin.es/estadisticas/ecvt/ecvt2010.

${ }^{6}$ Las variables responsables de la reducción de la muestra están relacionadas con las respuestas sobre la dificultad para solicitar días libres por motivos familiares y para ausentarse del trabajo para resolver asuntos particulares esporádicos.

7 La razón de re-escalar la variable satisfacción es porque existe poca variabilidad, y, generalmente, hay pocas observaciones en los niveles más bajos.
} 
- 2 para los trabajadores muy satisfechos (8, 9 y 10$)$

La ECVT proporciona también información sobre las características personales del trabajador y del empleo que ocupa. A continuación se detallan las variables que se han considerado como regresores en la estimación:

1. Características personales: edad, sexo, nacionalidad, nivel de estudios, composición familiar (número de hijos menores de 15 años, modalidad de hogar y si tienen dependientes a su cargo), tamaño del municipio de residencia y si está pluriempleado.

2. Variables que recogen la actividad que desarrolla la empresa (código CNAE-09).

3. Variables que recogen la ocupación o profesión del trabajador en su empleo principal (código CNO-94).

4. Variables relacionadas con la remuneración del trabajo: ingresos mensuales netos y si recibe ayudas sociales por parte de la empresa.

5. Variables relacionadas con el tiempo en el trabajo y tipo de contrato: contrato indefinido o temporal, si trabaja en el sector público, duración de la jornada laboral, frecuencia con la que prolonga la jornada, si trabaja los fines de semana, si trabaja por turnos, tiempo de desplazamiento al trabajo y tipo de jornada laboral.

6. Variables que recogen otros aspectos del trabajo: si trabaja en equipo, si tiene disponibilidad de movilidad de residencia, si tiene disponibilidad de viajar por motivos de trabajo, número de trabajadores de la empresa, si pertenece a alguna asociación profesional, si está afiliado a algún sindicato, si la empresa facilita la negociación colectiva y si está sujeto a algún convenio específico de regulación.

7. Variables relacionadas con la conciliación entre la vida laboral y familiar: tiempo dedicado a las tareas del hogar, dificultad para ausentarse y dificultad para solicitar días sin empleo y sueldo por motivos familiares, y como ha afectado o afectaría la maternidad/paternidad a la trayectoria profesional.

8. Variables regionales, que controlan las características de los mercados laborales regionales. 
34 Rico Belda /Revista de Economía Laboral 9 (2012), 30-45

Cuadro 1. Medias muestrales por género y nacionalidad

\begin{tabular}{|c|c|c|c|c|c|}
\hline & Muestra total & Mujeres & Hombres & Nativos & Inmigrantes \\
\hline \multicolumn{6}{|l|}{ Características Personales } \\
\hline \multirow[t]{2}{*}{ Edad } & 41,96 & 41,56 & 42,27 & 42,44 & 37,40 \\
\hline & $(10,38)$ & $(10,22)$ & $(10,51)$ & $(10,40)$ & $(9,07)$ \\
\hline \multirow[t]{2}{*}{ Antigüedad } & 11,04 & 10,22 & 11,72 & 11,77 & 4,23 \\
\hline & $(10,29)$ & $(9,85)$ & $(10,59)$ & $(10,46)$ & $(4,56)$ \\
\hline Nivel de estudios & 5,87 & 6,18 & 5,62 & 5,90 & 5,64 \\
\hline Hasta E. Primarios ${ }^{*}$ & 0,15 & 0,12 & 0,16 & 0,14 & 0,19 \\
\hline Secundarios ${ }^{*}$ & 0,21 & 0,18 & 0,23 & 0,20 & 0,25 \\
\hline$F P 1^{*}$ & 0,11 & 0,11 & 0,12 & 0,12 & 0,08 \\
\hline$F P 2^{*}$ & 0,11 & 0,11 & 0,12 & 0,12 & 0,07 \\
\hline Bachiller* & 0,13 & 0,14 & 0,12 & 0,13 & 0,21 \\
\hline Universitarios Medios ${ }^{*}$ & 0,12 & 0,17 & 0,09 & 0,13 & 0,09 \\
\hline Universitarios Superiores ${ }^{*}$ & 0,15 & 0,17 & 0,14 & 0,16 & 0,12 \\
\hline \multicolumn{6}{|l|}{ Modalidad de hogar } \\
\hline Solos ${ }^{*}$ & 0,10 & 0,10 & 0,09 & 0,10 & 0,10 \\
\hline Familia monoparental ${ }^{*}$ & 0,05 & 0,10 & 0,01 & 0,05 & 0,08 \\
\hline Con pareja sin hijos ${ }^{*}$ & 0,17 & 0,18 & 0,17 & 0,17 & 0,16 \\
\hline Con pareja con hijos* & 0,50 & 0,43 & 0,56 & 0,50 & 0,49 \\
\hline Otros $^{2}$ & 0,18 & 0,19 & 0,17 & 0,17 & 0,18 \\
\hline Número hijos menores de 15 años & 0,54 & 0,50 & 0,58 & 0,52 & 0,74 \\
\hline Dependientes a su cargo* & 0,07 & 0,07 & 0,07 & 0,07 & 0,03 \\
\hline Pluriempleo* & 0,03 & 0,03 & 0,03 & 0,03 & 0,04 \\
\hline \multicolumn{6}{|l|}{ Remuneración trabajo } \\
\hline Ingresos netos mesuales & 3,54 & 3,11 & 3,88 & 3,61 & 2,89 \\
\hline Recibe ayudas por parte empresa* & 0,49 & 0,47 & 0,49 & 0,50 & 0,34 \\
\hline \multicolumn{6}{|l|}{ Tipo de trabajo $y$ horas trabajo } \\
\hline Tipo de contrato* & 0,79 & 0,78 & 0,80 & 0,81 & 0,65 \\
\hline Jornada continuada* & 0,59 & 0,67 & 0,53 & 0,60 & 0,53 \\
\hline Jornada completa ${ }^{*}$ & 0,87 & 0,78 & 0,94 & 0,87 & 0,83 \\
\hline No trabaja en fin de semana* & 0,51 & 0,54 & 0,48 & 0,52 & 0,38 \\
\hline Trabaja por turnos * & 0,21 & 0,21 & 0,20 & 0,21 & 0,17 \\
\hline \multirow[t]{2}{*}{ Tiempo al trabajo } & 1,72 & 1,71 & 1,74 & 1,70 & 1,93 \\
\hline & $(0,97)$ & $(0,94)$ & $(0,99)$ & $(0,95)$ & $(1,11)$ \\
\hline \multirow[t]{2}{*}{ Duración jornada } & 38,74 & 36,04 & 40,93 & 38,73 & 38,82 \\
\hline & $(8,61)$ & $(9,47)$ & $(7,13)$ & $(8,50)$ & $(9,67)$ \\
\hline \multicolumn{6}{|l|}{ Frecuencia prolongación jornada } \\
\hline Nunca ${ }^{*}$ & 0,33 & 0,37 & 0,29 & 0,33 & 0,33 \\
\hline Ocasionalmente ${ }^{*}$ & 0,47 & 0,44 & 0,49 & 0,47 & 0,47 \\
\hline Mitad de los dias" & 0,09 & 0,09 & 0,09 & 0,09 & 0,08 \\
\hline Siempre ${ }^{*}$ & 0,12 & 0,11 & 0,13 & 0,12 & 0,12 \\
\hline Trabaja en el sector público & 0,25 & 0,31 & 0,20 & 0,26 & 0,10 \\
\hline \multicolumn{6}{|l|}{ Otros aspecto del trabjo } \\
\hline Trabaja en equipo* & 0,81 & 0,80 & 0,82 & 0,82 & 0,71 \\
\hline Movilidad ${ }^{*}$ & 0,13 & 0,10 & 0,16 & 0,13 & 0,08 \\
\hline Viaja por motivos de trabajo* & 0,37 & 0,28 & 0,45 & 0,39 & 0,27 \\
\hline Sujeto a negociacion colectiva ${ }^{*}$ & 0,51 & 0,49 & 0,53 & 0,54 & 0,26 \\
\hline Sujeto a convenio específico ${ }^{*}$ & 0,39 & 0,38 & 0,41 & 0,42 & 0,20 \\
\hline Afiliación a un sindicato* & 0,21 & 0,19 & 0,23 & 0,23 & 0,07 \\
\hline Asociación profesional* & 0,09 & 0,09 & 0,09 & 0,10 & 0,04 \\
\hline \multirow[t]{2}{*}{ Trabajadores empresa } & 3,72 & 3,76 & 3,69 & 3,86 & 3,04 \\
\hline & $(1,22)$ & $(1,26)$ & $(1,18)$ & $(1,15)$ & $(1,19)$ \\
\hline \multirow[t]{2}{*}{ Trabajadores centro } & 3,01 & 2,99 & 3,03 & 3,06 & 2,54 \\
\hline & $(1,09)$ & $(1,12)$ & $(1,07)$ & $(1,09)$ & $(0,99)$ \\
\hline \multicolumn{6}{|l|}{ Conciliación } \\
\hline Tiempo tareas hogar & 1,62 & 2,16 & 1,18 & 1,62 & 1,57 \\
\hline & $(1,33)$ & $(1,33)$ & $(1,17)$ & $(1,33)$ & $(1,33)$ \\
\hline Dificultad dias libres & 3,54 & 3,49 & 3,58 & 3,45 & 4,40 \\
\hline Dificultad para ausentarse & 3,30 & 3,35 & 3,26 & 3,22 & 4,04 \\
\hline Maternidadpaternidad influiría positivamente* & 0,12 & 0,08 & 0,16 & 0,12 & 0,11 \\
\hline Maternidadparternidad influiría negativamente & 0,17 & 0,25 & 0,10 & 0,16 & 0,23 \\
\hline Satisfacción conciliación & 6,89 & 6,79 & 6,97 & 6,93 & 6,58 \\
\hline Satisfacción laboral & 7,43 & 7,46 & 7,41 & 7,45 & 7,26 \\
\hline Contraste igualdad medias (p-value $)^{* *}$ & & eres-Hombre: & Nat & os-Inmigr & \\
\hline Satisfacción conciliación & & 0,00 & & 0,00 & \\
\hline Satisfacción laboral & & 0,21 & & 0,01 & \\
\hline
\end{tabular}


El Cuadro 1 recoge la media muestral de las variables consideradas y, con la intención de no ser excesivamente exhaustivo, se va a comentar las diferencias y similitudes observadas, entre hombre y mujeres y entre nativos e inmigrantes, que resultan más interesantes. Así, se observa que hombres y mujeres presentan una edad media muy similar, alrededor de los 42 años, aunque la antigüedad de los hombres, no obstante, es ligeramente superior a la de las mujeres. Por otro lado, las mujeres presentan, en media, mayor formación que los hombres ${ }^{8}$, y el porcentaje de mujeres con estudios universitarios es superior al de los hombres. No obstante, a pesar de la mayor formación de las mujeres, sus ingresos mensuales medios son inferiores al de los varones ${ }^{9}$.

En el caso de los inmigrantes, la edad media de éstos es menor a la de los trabajadores nativos, a la vez que llevan bastante menos años trabajando. Respecto a la remuneración del trabajo, los asalariados extranjeros perciben unos ingresos netos mensuales medios inferiores al de los asalariados españoles, mientras que el porcentaje de trabajadores inmigrantes que reciben ayudas sociales por parte de la empresa es mucho menor que el de los trabajadores nativos.

Con respecto al tipo de trabajo y horas de trabajo, alrededor del $80 \%$ de los asalariados tienen un contrato de duración indefinida, porcentaje que cae hasta el $65 \%$ en el caso de los trabajadores inmigrantes. Los hombres trabajan, en media, alrededor de cinco horas semanales más que las mujeres, no observándose diferencias por nacionalidad.

Analizando otros aspectos del trabajo, del Cuadro 1 se observa que sólo el $28 \%$ de las mujeres encuestadas declaran viajar por motivos de trabajo, frente al 45\% de los hombres, observándose pequeñas diferencias entre trabajadores inmigrantes y españoles. Asimismo, del Cuadro 1 se deduce que tan sólo el $10 \%$ de los asalariados pertenece a una asociación profesional y solo alrededor del $20 \%$ está afiliado a un sindicato. En el caso de los inmigrantes, estos porcentajes se reducen al $4 \%$ y $7 \%$, respectivamente.

En relación a las variables relacionadas con la conciliación, éstas indican que las mujeres dedican mucho más tiempo a las tareas

\footnotetext{
8 La variable nivel de estudios, obtenida directamente de la ECVT, es una variable ordinal que considera nueve niveles educativos de forma ascendente.

9 Los ingresos netos mensuales es una variable ordinal que toma nueve valores, según la ECVT de 2010: 1. Hasta 600 euros, 2. De 600 a 1.000 euros, 3. De 1.001 a 1.200, 4. De 1.201 a $1.600,5$. De 1.601 a 2.100, 6. De 2.101 a 3.000, 7. De 3.001 a 4.500, 8. De 4.501 a $6.000,9$. Más de 6.000 euros.
} 
domésticas que los hombres ${ }^{10}$, mientras que no se observan diferencias entre los trabajadores inmigrantes y nativos. Con respecto a la dificultad para solicitar días sin empleo y sueldo por motivos familiares y a la dificultad para ausentarse, estos ítems ${ }^{11}$ presentan valores medios reducidos, siendo mayor en el caso de los trabajadores inmigrantes. Finalmente, y por lo que respecta a las expectativas sobre como la maternidad/paternidad del trabajador ha influido/influiría en su carrera profesional, el porcentaje de trabajadores que piensan que ha influido/influiría no es muy elevado, observándose que en el caso de las mujeres la opinión generalizada es que les afectaría negativamente.

Por lo que se refiere a la satisfacción con la conciliación, el nivel medio de ésta es menor a la satisfacción laboral ${ }^{12}$, no superando el nivel de 7 para ningún colectivo. El contraste de igualdad de medias rechaza la hipótesis nula de igualdad, tanto entre hombres y mujeres, como entre nativos e inmigrantes. En la satisfacción por género la diferencia es desfavorable para las mujeres, mientras que por nacionalidad la comparación es desfavorable para los asalariados inmigrantes.

\section{Resultados empíricos}

En el apartado anterior se evidenció que los asalariados mujeres e inmigrantes se muestran menos satisfechos con la conciliación laboral y familiar que los trabajadores hombres y los nativos, respectivamente. Asimismo, se observó diferencias, entre estos colectivos, en algunas de las características personales y laborales. Ahora se va a comprobar si las diferencias en la satisfacción con la conciliación vienen explicadas por las características personales y/o laborales de los trabajadores.

No obstante, antes de analizar los resultados empíricos del modelo estimado, se quiere considerar dos cuestiones fundamentales a tener en cuenta:

Una de ellas hace referencia a la naturaleza cardinal u ordinal de la satisfacción. En economía, generalmente, se supone que la satisfacción

\footnotetext{
${ }^{10}$ Esta evidencia se presenta en muchos otros trabajos como queda recogido en Gracia y Bellani (2010).

11 Son variables ordinales que toman valores de una escala del 0 al 10 , donde 0 es ninguna dificultad y 10 mucha dificultad.

12 Un poco más del 55\% de los asalariados declaran una satisfacción laboral igual o superior a 8 (sobre 10), mientras que con la satisfacción con la conciliación, este porcentaje es del $42,3 \%$, reduciéndose hasta el $34,7 \%$ en el caso de los inmigrantes.
} 
es ordinal ${ }^{13}$ y a través de los modelos de respuesta múltiple ordenada se considera que la satisfacción es un indicador ordinal de una variable latente inobservable, que se interpreta como el bienestar esperado que reporta el trabajo ${ }^{14}$.

\section{Cuadro 2. Estimación de la satisfacción con la conciliación laboral y familiar. Modelo probit ordenado.}

\begin{tabular}{|c|c|c|c|c|c|c|c|}
\hline \multicolumn{6}{|c|}{ Efectos } & \multicolumn{2}{|r|}{ Efectos } \\
\hline & & Coeficientes & Marginales & & & Coeficientes & Marginales \\
\hline \multicolumn{2}{|l|}{ Hombre } & 0,057 & 0,023 & \multicolumn{2}{|c|}{ Viaja por motivos de trabajo } & 0,040 & 0,016 \\
\hline \multicolumn{2}{|l|}{ Inmigrante } & $-0,014$ & $-0,005$ & \multicolumn{2}{|l|}{ Trabaja en equipo } & 0,136 & 0,053 \\
\hline \multicolumn{2}{|l|}{ Edad } & 0,056 & $-0,022$ & \multicolumn{2}{|c|}{ Sujeto a negociación colectiva } & 0,080 & 0,032 \\
\hline \multicolumn{2}{|l|}{$\left(\operatorname{Edad}^{\wedge} 2\right) / 100$} & 0,070 & 0,024 & \multicolumn{2}{|c|}{ Sujeto a convenio específico } & $-0,130$ & $-0,051$ \\
\hline \multicolumn{2}{|l|}{ Antigüedad } & $-0,003 *$ & $-0,001$ & \multicolumn{2}{|l|}{ Trabajadores empresa } & $-0,006$ & $-0,003$ \\
\hline \multicolumn{2}{|c|}{ Hijos menores 15 años } & $-0,053$ & $-0,021$ & \multicolumn{2}{|l|}{ Afiliación a sindicato } & 0,031 & 0,012 \\
\hline \multicolumn{2}{|l|}{ Dependientes } & $-0,106^{*}$ & $-0,042$ & \multicolumn{2}{|l|}{ Asociación profesional } & $-0,062$ & $-0,025$ \\
\hline \multirow[t]{4}{*}{ Tamaño municipio } & De 10.001 a 50.000 hab. & $-0,108$ & $-0,042$ & \multirow[t]{8}{*}{ Ocupación: } & Dirección & 0,116 & 0,046 \\
\hline & De 50.001 a 100.000 hab. & $-0,108^{*}$ & $-0,043$ & & Administrativo & $-0,007$ & $-0,003$ \\
\hline & De 100.001 a 1.000 .000 hab. & $-0,136$ & $-0,053$ & & Agricultura & 0,341 & 0,136 \\
\hline & Más de 1.000 .000 hab. & $-0,327$ & $-0,124$ & & Artesano & 0,213 & 0,085 \\
\hline \multirow[t]{4}{*}{ Modalidad de hogar: } & Familia monoparental & $-0,098$ & $-0,038$ & & Montador & 0,043 & 0,017 \\
\hline & Con pareja sin hijos & 0,012 & 0,005 & & Técnico & 0,167 & 0,067 \\
\hline & Con pareja con hijos & $-0,081$ & $-0,032$ & & Técnico de apoyo & 0,066 & 0,026 \\
\hline & Otros & 0,001 & 0,000 & & No cualificado & 0,083 & 0,033 \\
\hline \multirow[t]{6}{*}{ Educación: } & Secundaria & $-0,086$ & $-0,034$ & \multirow[t]{11}{*}{ Actividad empresarial: } & Agricultura & $-0,124$ & $-0,048$ \\
\hline & FP1 & $-0,158$ & $-0,039$ & & Construcción & $-0,136$ & $-0,053$ \\
\hline & $\mathrm{FP} 2$ & $-0,098$ & $-0,030$ & & Comercio & $-0,002$ & $-0,001$ \\
\hline & Bachiller & $-0,075$ & $-0,061$ & & Transporte & 0,058 & 0,023 \\
\hline & Univ. Medio & $-0,202$ & $-0,078$ & & Hosteleria & 0,292 & 0,116 \\
\hline & Univ. Superior & $-0,157$ & $-0,061$ & & Información/Finanzas & $-0,065$ & $-0,025$ \\
\hline \multicolumn{2}{|c|}{$\begin{array}{l}\text { Pluriempleo } \\
\text { In resos netos mesuales }\end{array}$} & $-0,307$ & $-0,117$ & & AAPP & 0,153 & 0,061 \\
\hline \multirow{2}{*}{\multicolumn{2}{|c|}{$\begin{array}{l}\text { Ingresos netos mesuales } \\
\text { Recibe ayudas por parte empresa }\end{array}$}} & 0,023 & 0,009 & & Act. Profesionales & $-0,012$ & $-0,005$ \\
\hline & & 0,132 & 0,052 & & Educación & 0,228 & 0,116 \\
\hline \multicolumn{2}{|l|}{ Duración jornada } & $-0,014$ & $-0,006$ & & Sanidad & 0,044 & 0,017 \\
\hline \multicolumn{2}{|c|}{ Prolonga jornada siempre } & $-0,336$ & $-0,127$ & & Otras actividades & 0,033 & 0,013 \\
\hline Prolonga jornada mi & & $-0,341$ & $-0,129$ & Tiempo tareas hogar & & $-0,005$ & $-0,002$ \\
\hline Prolonga jornada oc: & onalmente & $-0,118$ & $-0,046$ & Dificultad días libres & & $-0,016$ & $-0,011$ \\
\hline Trabaja en el sector & blico & $-0,035$ & $-0,014$ & Dificultad para ausents & arse & $-0,017$ & $-0,011$ \\
\hline Tipo de contrato & & 0,066 & 0,026 & Maternidad/Paternidac & d influiría positivamente & 0,234 & 0,093 \\
\hline Jornada continuada & & 0,138 & 0,054 & Maternidad/Paternidad & d influiría negativamente & $-0,451$ & $-0,167$ \\
\hline Jornada completa & & $-0,117$ & $-0,046$ & Región: & sur & 0,094 & 0,037 \\
\hline Trabaja fines de sem & & $-0,197$ & $-0,076$ & & Noroeste & 0,080 & 0,032 \\
\hline Trabaja por turnos & & $-0,068$ & $-0,027$ & & Nordeste & 0,005 & 0,002 \\
\hline Tiempo al trabajo & & $-0,105$ & $-0,041$ & & Este & 0,059 & 0,023 \\
\hline Movilidad & & $-0,072$ & $-0,028$ & & Centro & $0,142 *$ & 0,057 \\
\hline Número de observac & & 5594 & & Estadístico razón de ve & erosimilitud: & 756,721 & \\
\hline Pseudo R-cuadrado: & & 0,073 & & R-cuadrado de predicci & & 55,703 & \\
\hline Log. Likelihood: & & $-4796,731$ & & & & & \\
\hline
\end{tabular}

La otra cuestión a considerar es que los datos transversales conllevan algunas limitaciones que conviene no olvidar. Una de estas

13 Los psicólogos y sociólogos, generalmente, interpretan la satisfacción como una medida cardinal, de forma que las diferencias entre niveles de satisfacción tienen un valor específico (ver Ferrer-i-Carbonell y Frijters (2004)).

${ }^{14}$ Los modelos de respuesta múltiple ordenada permiten obtener la probabilidad para cada una de los niveles de satisfacción considerados, así como los efectos marginales que las variables explicativas tienen sobre cada una de las probabilidades. 
limitaciones está relacionada con la posibilidad de que presenten heterocedasticidad no observada, relacionada, fundamentalmente, con factores de la personalidad ${ }^{15}$. Por otro lado, los datos transversales suelen presentar sesgos de selección, esto es, sesgos que se producen en el momento de seleccionar los participantes en el estudio. En este trabajo los datos proceden de la ECVT de 2010, encuesta que va dirigida a una muestra representativa de los trabajadores españoles, obtenida de forma aleatoria de acuerdo a un muestreo trietápico estratificado.

El Cuadro 2 recoge los resultados de la estimación del modelo probit ordenado $^{16}$ de la satisfacción con la conciliación laboral y familiar de los asalariados para la muestra seleccionada. En este modelo se ha incluido una variable que recoge el género de cada trabajador, así como una variable que recoge su nacionalidad.

Siguiendo a Álvarez (2005), dada la no linealidad del modelo estimado, resulta conveniente evaluar el impacto que sobre la variable dependiente ejerce cada una de las variables explicativas. Para ello se ha calculado la probabilidad de estar muy satisfecho para un individuo de referencia. Este individuo es aquel en el que todas las variables explicativas están fijadas en el valor 0 , salvo la edad, la duración de la jornada y el tiempo al trabajo en las que el valor de referencia es la media muestral. Asimismo, para las variables ordinales ${ }^{17}$ la media se redondea a su valor entero más próximo. Una vez obtenida la probabilidad, se ha calculado el impacto de un cambio unitario en cada una de las variables explicativas, manteniendo todas las demás constantes. Este impacto aparece recogido en la segunda columna del Cuadro 2.

Analizando los coeficientes estimados, se observa que hombres y mujeres presentan el mismo nivel de satisfacción, ceteris paribus. Asimismo, el coeficiente de la variable que recoge la nacionalidad del trabajador tampoco resulta ser significativo, por lo que tampoco se evidencia la existencia de un efecto diferencial por nacionalidad en la satisfacción con la conciliación. Con respecto a la edad, se ha obtenido una

\footnotetext{
15 La solución propuesta por algunos autores es la utilización de datos de panel que permiten tener en cuenta las características, persistentes y no observables, del individuo y, por tanto, corregir los resultados por los efectos de estos factores. En este trabajo al estimar el modelo se ha considerado las varianzas-covarianzas de los estimadores consistentes con heterocedasticidad.

16 El modelo logit ordenado presenta una bondad de ajuste ligeramente inferior al del modelo probit, finalmente seleccionado.

17 Ingresos mensuales netos, dificultad para solicitar días libres y dificultad para ausentarse.
} 
relación convexa entre la edad y la satisfacción ${ }^{18}$, de forma que el mínimo de dicha relación se sitúa en algo menos de los 40 años. Parece razonable este resultado puesto que a partir de esa edad, generalmente los hijos suelen alcanzar una edad que necesitan menos atención y, por tanto, es más fácil conciliar, aumentando con ello la satisfacción con la conciliación. El número de hijos menores de 15 años y tener a su cargo personas dependientes influye negativamente en la satisfacción del trabajador con la conciliación. La satisfacción con la conciliación se reduce con el tamaño del municipio de residencia, observándose un elevado efecto diferencial en los municipios de más de 1,000.000 de habitantes, lo que indicaría que en las áreas urbanas más reducidas es más fácil conciliar la vida laboral y familiar que en las grandes urbes. Con respecto a la modalidad de hogar, la variable de referencia es vivir solo, pero no se evidencia efectos diferenciales significativos de los trabajadores que presentan otra modalidad de familia.

En cuanto a la educación, la variable de referencia es tener hasta estudios primarios, de tal forma que se observa que la satisfacción con la conciliación es menor en los trabajadores con estudios superiores. Esto puede venir explicado por el hecho de que generalmente el mayor nivel de estudios implica empleos de mayor responsabilidad y ello podría dificultar la conciliación, repercutiendo finalmente en el nivel de satisfacción. Por otra parte, tener más de un puesto de trabajo conlleva, como cabría esperar, un efecto diferencial negativo en la satisfacción con la conciliación.

Respecto a las características laborales, se observa que el nivel de satisfacción con la conciliación no viene determinado por los ingresos netos mensuales ${ }^{19}$, pero sí que se observa un efecto diferencial positivo en la satisfacción con la conciliación en favor de los asalariados que reciben ayudas sociales por parte de la empresa, frente a los que no reciben. Por otro lado, se observa que la prolongación de la jornada laboral reduce el bienestar del trabajador con la conciliación. No se obtiene evidencia de que los trabajadores del sector público presenten niveles de satisfacción con la conciliación diferentes a los trabajadores del sector privado.

El tipo de contrato no tiene efecto sobre la satisfacción con la conciliación, pero si el tipo de jornada. De esta forma, tener jornada partida y/o completa implica un menor nivel de satisfacción con la

18 Gamero (2010) también muestra una relación en forma de U entre edad y satisfacción laboral.

19 Se consideró la posibilidad de introducir esta variable elaborando variables dicotómicas para cada uno de los segmentos considerados en la ECVT, así como utilizar la marca de clase para cada intervalo, pero, dado que no mejoraban los resultados del modelo, se desestimaron. 
conciliación de los trabajadores, frente a tener jornada continuada y/o parcial. Asimismo, los trabajadores que declaran trabajar los fines de semana presentarían niveles de satisfacción con la conciliación más reducidos, que los que no lo hacen. Finalmente, el tiempo que se tarda en llegar al trabajo afecta negativamente al nivel de satisfacción con la conciliación.

Con respecto a los coeficientes estimados de otros aspectos laborales, los trabajadores que están sujetos a movilidad por parte de la empresa y los que viajan por motivos de trabajo no presentan niveles de satisfacción con la conciliación diferentes a los que no lo hacen. Sin embargo, los trabajadores que trabajan en equipo presentan niveles de satisfacción con la conciliación más elevados que los trabajadores que no lo hacen. Esto puede deberse a que en momentos puntuales el equipo podría facilitar la posibilidad de ausentarse, al poder suplir la falta de algún miembro sin mayor problema. Por otro lado, no se evidencia la existencia de efectos diferenciales en la satisfacción con la conciliación de los trabajadores afiliados a un sindicato o que pertenecen a una asociación profesional. Por su parte, el nivel de satisfacción con la conciliación de los trabajadores de empresas que facilitan la negociación colectiva es mayor que el de los trabajadores de empresas que no lo hacen. Asimismo, los asalariados no sujetos a convenio o estatuto específico de regulación se muestran más satisfechos con la conciliación que los que se ven afectados. Finalmente, el tamaño de la empresa, utilizando como proxy el número de trabajadores, no influye en el nivel de bienestar con la conciliación de sus asalariados.

Analizando la ocupación de los trabajadores, se observa un diferencial positivo en la satisfacción con la conciliación de los trabajadores cuya ocupación es la agricultura, la artesanía o ser técnico, respecto de la categoría de referencia que es ocuparse en el comercio. En relación a la actividad económica a la que se dedica la empresa, los trabajadores de empresas cuya actividad es la construcción presentan niveles de satisfacción con la conciliación menores que los trabajadores de la categoría de referencia, que son los que trabajan en la industria. Por su parte, los trabajadores de empresas dedicadas a la hostelería, la educación y a la Administraciones Públicas presentan niveles superiores de bienestar con la conciliación a los de la categoría de referencia.

En relación a las variables relacionadas con la conciliación, la dificultad para solicitar días libres sin sueldo o para ausentarse tienen efectos negativos en la satisfacción con la conciliación, mientras que las horas dedicadas al hogar no influyen en la satisfacción. Por su parte, se observa que las expectativas positivas de la maternidad/paternidad sobre la carrera profesional aumentan la satisfacción con la conciliación, 
mientras que en el caso de que las expectativas sean negativas, éstas influyen negativamente sobre el bienestar con la conciliación y además su efecto diferencial es mayor al que tienen las expectativas positivas.

Como se ha visto, considerando las características personales y laborales de los asalariados, se ha obtenido que no existen diferencias significativas ni por nacionalidad ni por género en el nivel de satisfacción con la conciliación. Siguiendo a Gamero (2010), se va a analizar las variables de control responsables de no evidenciar diferencias de género y de nacionalidad.

El cuadro 3 recoge los coeficientes estimados para las variables que recogen el género y la nacionalidad del asalariado para distintas especificaciones consideradas. En primer lugar, únicamente se han incluido estas dos variables, obteniéndose que ambas son significativas y presentan los signos esperados. Este resultado evidencia que, cuando no se introduce ninguna variable de control, existen efectos diferenciales en la satisfacción con la conciliación en favor de los hombres y de los trabajadores nativos. A partir de esta especificación, se ha ido añadiendo, alternativamente, los distintos bloques de variables consideradas, con el objetivo de comprobar qué factores son los responsables de la pérdida de la significatividad en la especificación final.

\section{Cuadro 3. Resultados de diferentes especificaciones para la satisfacción con la conciliación laboral y familiar.}

\begin{tabular}{lcccc}
\hline & $\begin{array}{c}\text { Coeficiente } \\
\text { Hombre }\end{array}$ & $\begin{array}{c}\text { Coeficiente } \\
\text { Inmigrante }\end{array}$ & $\begin{array}{c}\text { Predicciones } \\
\text { Pseudo-R2 correctas (\%) }\end{array}$ \\
\hline 1. Ninguna variable de control & $\mathbf{0 , 0 6 6}$ & $\mathbf{- 0 , 1 4 4}$ & 0,001 & 48,83 \\
2. 1+Características personales & 0,036 & $-0,067$ & 0,015 & 50,19 \\
3. 1+Remuneración trabajo & $\mathbf{0 , 1 0 2}$ & $\mathbf{- 0 , 1 5 6}$ & 0,004 & 50,19 \\
4. 1+Horas trabajo y tipo contrato & $\mathbf{0 , 1 8 5}$ & $-0,074$ & 0,029 & 51,36 \\
5. 1+Actividad empresarial & $\mathbf{0 , 1 0 9}$ & $\mathbf{- 0 , 1 1 4}$ & 0,007 & 49,95 \\
6. 1+Ocupación & $\mathbf{0 , 0 5 2 ^ { * }}$ & $\mathbf{- 0 , 1 7 3}$ & 0,003 & 49,52 \\
7. 1+Otros aspectos laborales & $\mathbf{0 , 0 7 6}$ & $\mathbf{- 0 , 1 3 9}$ & 0,005 & 49,84 \\
8. 1+Variables conciliación & 0,002 & $\mathbf{- 0 , 1 1 7}$ & 0,025 & 53,35 \\
9. Especificación final & 0,057 & $-0,014$ & 0,073 & 55,70 \\
\hline
\end{tabular}

Fuente: Elaboración propia a partir de ECVT 2010.

Nota: Los parametros en negrita son estadísticamente significativos.

* Significativo al $10 \%$.

De los resultados mostrados en el Cuadro 3 se puede comprobar que la variable indicadora del género pierde su significatividad cuando se añaden las características personales y las variables relacionadas con la conciliación. Por su parte, las variables causantes de la pérdida de la significatividad de la variable indicadora de la nacionalidad del trabajador 
son, al igual que en el género, las características personales, pero aquí también juegan un papel importante las variables relacionadas con el tiempo en el trabajo.

\section{Conclusiones}

En este trabajo se ha analizado los factores que explican el nivel de satisfacción con la conciliación laboral y familiar de los asalariados en España, haciendo especial hincapié en las diferencias de género y de nacionalidad. Para ello se ha utilizado la ECVT 2010 que, por primera vez, desde que viene realizándose, ha requerido del entrevistado que declarase su nivel de satisfacción con la conciliación laboral y familiar.

La estimación del modelo probit de respuesta ordenada indica que, después de controlar las características personales y laborales de los asalariados, no se evidencia la existencia de efectos diferenciales ni por razón de sexo ni de nacionalidad. No obstante, sin factores de control, los hombres presentan un nivel de satisfacción con la conciliación mayor que las mujeres, mientras que los asalariados inmigrantes presentan un nivel de satisfacción con la conciliación mucho menor que los asalariados nativos.

Un análisis más detallado ha permitido conocer los factores explicativos que causan la pérdida de significatividad de las variables indicadoras del género y de la nacionalidad de los asalariados. De esta forma, en el caso de la variable indicadora de género son las características personales y las variables relacionadas con la conciliación las causantes de su pérdida de poder predictivo. En el caso de la variable que recoge la nacionalidad, han sido las características personales y las variables relacionadas con el tiempo en el trabajo.

De los resultados obtenidos se pueden concluir que la satisfacción con la conciliación se ve positivamente influida si los trabajadores reciben ayudas sociales por parte de las empresas, si trabajan en equipo y si las empresas facilitan la negociación colectiva. Por su parte, el nivel de satisfacción con la conciliación se ve influido negativamente por la edad hasta los 40 años, por el número de hijos, si se tienen dependientes a cargo, el tamaño del municipio, el nivel de educación y si se está pluriempleado. Asimismo, la jornada partida, la jornada completa, la dificultad para solicitar días libres y para ausentarse, el tiempo que se tarda en llegar al trabajo, la prolongación de la jornada laboral y trabajar los fines de semana también afectan negativamente en la satisfacción con la conciliación de los asalariados españoles. 
De estos resultados varias serían las medidas de política empresarial y/o social que podrían llevarse a cabo con el objetivo de aumentar la satisfacción con la conciliación de los asalariados en España. Por ejemplo, las empresas interesadas en incrementar el bienestar de sus trabajadores, y con ello beneficiarse ellas, a medio y largo plazo, con mejoras en la productividad y clima empresarial, deberían de fomentar el trabajo en equipo, proporcionar ayudas sociales a sus trabajadores, como forma de remuneración, y facilitar la negociación colectiva. Asimismo, dado que el tiempo que se tarda en llegar al trabajo tiene un efecto negativo en el bienestar laboral, las empresas deberían de contratar mano de obra local ${ }^{20}$. Por otro lado, deberían de tratar de no prolongar las jornadas laborales y flexibilizarlas, promoviendo el trabajo en el domicilio, siempre que el trabajo lo permita, y fomentando jornadas continuas y parciales, que permitan al trabajador planificar su vida laboral y personal sin afectar al rendimiento del trabajo. Finalmente, también aumentaría el bienestar de sus trabajadores, si la empresa facilitase que puedan solicitar días libres sin sueldo por motivos familiares $y$ ausentarse esporádicamente del trabajo para solucionar asuntos personales.

Con respecto a la política social, varias son las medidas que ayudarían a hacer de la conciliación una realidad y mejorar con ello la satisfacción de los trabajadores con la conciliación entre su vida laboral y familiar:

1. Mayor apoyo y protección a la familia en el cuidado de los hijos y de las personas mayores. El gasto público en familias en España es más reducido que en otros países europeos, tales como Alemania y países nórdicos, donde conciliar es más fácil para los trabajadores $^{21}$.

2. Ampliar las medidas de bajas laborales por maternidad y paternidad que todavía son mínimas y bastantes inflexibles ${ }^{22}$.

3. Adecuar los horarios laborales y escolares para garantizar la conciliación de los trabajadores con hijos ${ }^{23}$.

\footnotetext{
${ }^{20}$ En este sentido, Mercadona, una de las empresas españolas que destaca, entre otras cosas, por facilitar la conciliación de sus trabajadores, utiliza en sus supermercados mano de obra mayoritariamente local.

21 UGT (2010) y Gracia y Bellani (2010).

22 Gracia y Bellani (2010).

${ }^{23}$ UGT (2010) evidencia que España no ha adaptado ni las relaciones laborales ni los sistemas educativos a las nuevas necesidades sociales.
} 


\section{Bibliografía}

Álvarez G. (2005): Análisis empírico de los determinantes de la satisfacción laboral en España, Revista de Economía y Empresa, 52 y 53 (2 Época) pág. 105-118.

Cantera, L.M., E. Cubells, L. Martinez and J. M. Blanch (2009): Work, family and Gender. Elements for a theory of work-life balance, The Spanish Journal of Psychology, 12(2), pág. 641-647.

Chinchilla, N. y C. León (2010): Diez años de con conciliación en España (1999-2009), ICWF, Centro Internacional Trabajo y Familia; The Family Watch.

Drew, E., R. Emereck and E. Mahon (1998): Women, work and family in Europe, London and New York, Routledge.

European Commision (2005): Reconciliation of work and private life: A comparative review of thirty European countries, Luxembourg: Office for official publications of the European Communities.

European Commision (2009): Reconciliation between work, private and family life in the European Union, Eurostat.

Ferrer-i-Carbonell, A. y P. Frijters (2004): "How important is methodology for the estimates of the determinants of happiness?", Journal of Public Economics 89, pág. 997-1019.

Gamero, C. (2010): "Satisfacción laboral de los asalariados inmigrantes", Revista de Economía Aplicada 54 (Vol. XVIII), pág. 33-56.

Gracia, P. y D. Bellani (2010): Las políticas de conciliación en España y sus efectos: un análisis de las desigualdades de género en el trabajo del hogar y el empleo, Estudios de progreso 51, Fundación Alternativas.

IESE (2012): Efectos de la conciliación en el compromiso, la satisfacción y el salario emocional, Centro Internacional Trabajo y Familia, Universidad de Navarra. 
Lewis, J., M. Campbell and C. Huerta (2008): Patterns of paid and unpaid work in Western Europe: gender, commodification, preferences and the implications for policy, Journal of European Social Policy, 18(1), pág. 2137.

Moltó, M.L. (2005): Reconciliation of work and private life in Spain. Informe para el Expert Group on Gender Social Inclusion and Employment EGG-SIE.

Observatorio efr (2009): Absentismo laboral: el colesterol de la empresa, Defensor del Menor, Comunidad de Madrid.

Observatorio efr (2010): El compromiso en el siglo XXI se escribe con "c" de conciliación, Defensor del Menor, Comunidad de Madrid.

Observatorio efr (2011): Impacto de la conciliación de la vida familiar y laboral sobre el bienestar de la infancia, Defensor del Menor, Comunidad de Madrid.

Ponce, J.M. (2007): La conciliación entre la vida personal y la profesional: un reto para las empresas y sus empleados, Revista Empresa $y$ Humanismo, 10(1), pág. 181-208.

UGT (2010): Las medidas de conciliación de la vida personal, familiar y laboral: su inclusión en la negociación colectiva, Secretaría de Análisis y Estrategia, UGT-Madrid.

Vera-Martínez, J.J., M. P. Martín (2009): Conciliación de la vida familiar y laboral: Estrategias de afrontamiento y reparto de las obligaciones familiares en parejas de doble ingreso, Anales de psicología, 25(1), pág. 142-149. 